\title{
A new start for 'Intestinal Research' as an official journal of AOCC
}

\author{
Toshifumi Hibi \\ Department of Internal Medicine, Kitasato Institute Hospital, Kitasato University, Tokyo, Japan \\ Editor-in-Chief, Intestinal Research
}

On behalf of the Asian Organization for Crohn's and Colitis (AOCC), I am honored to announce that Intestinal Research (Intest Res) becomes the official journal of AOCC and joint publication of the Chinese Society of IBD (CSIBD), the Japanese Society for IBD (JSIBD), Korean Association for the Study of Intestinal Disease (KASID), and the Taiwan Society of IBD (TSIBD).

AOCC was founded in 2013 through the collaboration of China, Korea, and Japan to improve the care of patients with IBD in Asia. Since then, AOCC has been constructing a platform to conduct collaborative research in IBD in Asia. I believe that Intest Res will strengthen the network and collaborating research among Asian countries in this field. In this point, publication of Intest Res as the official journal of AOCC is a great milestone for the Asian IBD community.

The incidence and prevalence of IBD has been rapidly increasing in Asia during the last several decades, although the incidence in Asia is still lower than western countries. One of the reasons for the lower incidence may be the difference of genetic background. Changes of environmental factors such as diet, microbes or smoking might have led to the increase of the patients. Studies of clinical aspects such as diagnosis and treatment are now in progress. We now realize that there are some similarity and difference in clinical characteristics and response to treatment in Asian patients compared with Westerns. There remain many challenges in this region regarding diagnosis, treatment, and research of IBD, because there are various kinds of region-specific issues including lifestyle, healthcare system, genetics, and epidemiology. We need to share our information and experience on the care of IBD patients with the rest of world. To meet those demands, Intest Res will provide an excellent platform for Asian doctors to transmit their research to the world.

I sincerely believe that Intest Res will play an important role in our collaboration and evolution of AOCC and provide the chance of IBD specialist to hold their tight cooperation for future research of IBD. The advancement of Intest Res is the key to success of IBD research in Asia. I strongly encourage Asian doctors and investigators to actively contribute to our official journal, Intest Res, by submitting original articles and reviews.

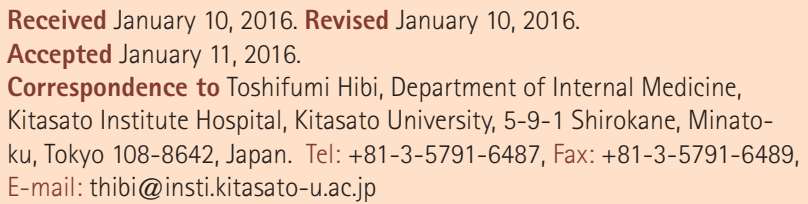

Financial support: None. Conflict of interest: None. 